\title{
Transfer of chirality from light to a Disperse Red 1 molecular glass surface
}

\author{
LeILA MAZAHeRI , ${ }^{1}$ OLIVIER LEBEL, ${ }^{2, *}$ AND JEAN-MICHEL NUNZI ${ }^{1,3^{*}}$ \\ ${ }^{1}$ Department of Physics, Engineering Physics \& Astronomy, Queen's university, Kingston K7L-3N6, Ontario, Canada \\ ${ }^{2}$ Department of chemistry and chemical Engineering, Royal Military College of Canada, Kingston K7K-7B4, Ontario, Canada \\ ${ }^{3}$ Department of chemistry, Queen's university, Kingston K7L-3N6, Ontario, Canada \\ *Corresponding authors: nunzijm@queensu.ca,Olivier.Lebel@rmc.ca
}

Received XX Month XXXX; revised XX Month, XXXX; accepted XX Month XXXX; posted XX Month XXXX (Doc. ID XXXXX); published XX Month XXXX

Chiral structures and materials interact with light in well-documented ways, but light can also interact with achiral materials to generate chirality by inscribing its asymmetric configuration on photoresponsive materials, such as azobenzene derivatives. While it is possible to thusly generate both bidimensional (2D) and tridimensional (3D) chirality, 2D chirality is especially attractive because of its non-reciprocity. Herein, 2D chirality is induced on the surface of a glass-forming Disperse Red 1 derivative by irradiation with a single laser beam, yielding crossed spontaneous surface relief gratings (SSRG) with different pitches. Azimuth rotations up to $10^{\circ}$ have been observed, and the absence of $3 D$ chirality has been confirmed. This method thus allows generating non-reciprocal planar chiral objects by a simple, single irradiation process on a thin film of a material that can easily be processed over large areas or onto small objects.

OCIS codes: (050.0050) Diffraction and gratings; (160.1190) Anisotropic optical materials; (090.2900) Optical storage materials.

http://dx.doi.org/10.1364/OL.99.099999

Chiral systems play an important role in nature and in our lives, from spiral galaxies to biologically active molecules such as DNA, amino acids and sugars [1]. Various aspects of these asymmetric systems have been studied in different fields of science [2-3]. In photonics, chiral configurations are utilized for manipulating electromagnetic waves for designing and improving the performance of photonic devices [4-6]. In addition to chiral structures that can be found in nature, recently there has been increasing interest to fabricate artificial chiral structures using methods such as electron beam lithography and chemical selfassembly [7-8]. These structures show promise for applications in technology including quantum computing [9], optical communication [10-11], negative refraction [12], polarization control, sensing, coupling, amplification, and optical switching [1315].
Chirality can be classified as two-dimensional (2D) or threedimensional (3D) according to non-reciprocity of the polarization rotation. An object or a system is $3 \mathrm{D}$ chiral if it is non superimposable with its mirror image by rotation in any direction. This definition applies for 2D chirality only if the rotation occurs in the plane. The helicity of a 3D chiral object is not directiondependent; it keeps its handedness even if it is flipped, given that the handedness of an object depends on the direction in which it is viewed [16]. A 3D chiral structure exhibits chiral-optical properties in the forms of circular birefringence and circular dichroism, which are identical for opposite wave propagation directions [17]. Circular birefringence is the consequence of a different propagation speed between left and right-handed circularly polarized light, and it manifests as an optical rotation of the plane of polarization. On the other hand, circular dichroism is a consequence of different extinction of left- and right-handed circularly polarized light, and is displayed experimentally as an ellipticity change [17]. In contrast, the helicity of a 2D chiral object is reversed for wave propagation in the opposite direction, as 2D chiral objects show planar symmetry. The chiral optical properties of a system can be either due to the individual chiral elements, or due to geometrical chirality, i.e. the chiral arrangement of nonchiral elements. Geometrical chirality vanishes if the individual elements are rearranged randomly, whereas molecular chirality will remain even if the molecules are randomly oriented in the plane [18]. Two-dimensional chirality is highly desirable in order to reduce the size of optical isolator, devices which are needed in optical communication systems [19-20]. Optical isolators usually require a magnet in order to benefit from the Faraday effect, which is non-reciprocal. The advent of efficient 2D-chiral materials and surfaces should permit to reduce optical isolation devices to just a thin-film coating [18-19].

There is some confusion in the literature regarding the source of optical activity for geometrical chiral structures. Optical activity cannot be a direct result of geometric 2D chirality [18]. Planar chiral-optical effects can be interpreted differently depending on the type of material used or the size of the structures. In the case of chiral metal nanostructures, the chiral-optical effects are related to surface plasmon resonance (SPRs) [21]. For metallic chiral 
microstructures (aligned gammadion), the optical activity is attributed to an induced current resulting from charge carriers which impulsion points in the direction opposite to the gammadion legs [22]. However, the same kind of chiral patterns have been inscribed on dielectric films, and similarly non reciprocal optical activities have been recorded [23], where induced current or SPR does not play any role. The important fact about planar chirality in dielectrics is that the change in polarization depends on the alignment and geometry of each individual element. If the structural components are aligned randomly, the structure is achiral. The dependence of optical rotation on the geometry of the planar chiral structure shows that chirality is not due to any chirality from the individual elements. Otherwise, the change in polarization of the output beam should depend on the excitation generated by the probe light rather than on the geometry itself. It was proposed that the optical activity of 2D chirality in dielectrics is a consequence of diffraction [23].

In a similar way that a chiral structure interacts with electromagnetic waves to induce circular birefringence and circular dichroism, electromagnetic waves can interact with materials to induce chirality. The chiral configuration of light can be recorded on a photoresponsive material, by inducing the formation of chiral structures in the material [24-25]. Azobenzene materials are suitable candidates to record chiral structures because they are sensitive to polarization, incidence angle, the phase and wavefront of the pump beams [26-27]. The generation of both 2D and 3D chirality has been reported on azobenzene materials using one simple holographic step [24-26,28]. While most azobenzene derivatives are achiral, chiral structures can be inscribed on them as a result of orientation and mass movement. 3D chirality is related to the helical orientation of the optical axis of an azo thin film. When the azo film is irradiated with a polarized beam, azo molecules on the surface isomerize and reorient with respect to the polarization of the light, then the optical axis of the top layer will rotate light polarization, which will in turn induce a new rotated optical axis in the layer underneath. As a result, the overall orientation of the optical axis will be helical, and therefore optically active.

Under certain experimental conditions, planar chiral structures can also be inscribed on azo thin films [25]. Irradiation of a thin film of azobenzene-containing material with an interference pattern of polarized laser beams results in the formation of surface relief gratings (SRGs) [28-29]. SRGs can also be inscribed using single beam exposure, where no external light pattern is applied and thus the structures form spontaneously [30-31]. The profile of these spontaneous surface relief grating (SSRG) depends on the polarization and incidence angle of the writing beam. The azo film acts as a data storage media, and information from the laser, such as wavelength, polarization and irradiation angle, gets stored into the azo material. Light absorption by the azo molecules, which depends on the angle between the light polarization and the azo transition dipole moment, regulate the reorientation of the azo molecules and the mass movement direction. Under chiral experimental conditions, the SSRG thusly formed may be chiral. In this work, both enantiomers of chiral SSRGs were inscribed on a Disperse Red 1 (DR1) azo glass-forming derivative (DR1-glass) [32]. Using a pump beam with different polarization states, distinct chiral structures were inscribed with a diffraction efficiency of $1 \%$, in the shape of chevron-like pavements. To identify 2D and 3D chiral properties, the optical activity of the gratings was studied for opposite probe beam propagation directions and for the mirror image gratings. In addition, the dependence of the chiral-optical properties of the SSRGs on sample thickness was studied. Planar chiral-optical effects showed to be dominant. Strong optical rotation reaching up to $10^{\circ}$ could be recorded for the SSRGs inscribed using circularly polarized light (CPL). The origin of the optical activity manifested by the SSRGs is attributed to a competition between reorientation of the azo molecules in the samples and the diffractive properties of the gratings under the Wood-anomaly conditions.

DR1-glass was prepared and thin films were deposited according to literature procedures [33,34]. SSRGs was inscribed using same setup as in reference [34]. Formation of SSRG was monitored by atomic force microscopy (AFM). The optical activity of the samples was monitored using a He-Ne laser at normal irradiation. The sample was set between a polarizer and an analyzer (see Figure 7 in [25]). In the absence of the sample, the transmitted light of the analyzer was recorded for different incidence polarizations. For each incoming polarization direction, the extinction of the analyzer was monitored for calibration. The sample was then inserted into the setup and the change in polarization between the transmitted beam for zero order and first order diffraction, and the incoming beam was recorded. The polarization state of the transmitted beam is described in terms of the geometrical parameters of the polarization ellipse: the orientation angle (azimuth angle) refers to the angle between the major axis of the ellipse and the $\mathrm{x}$ axis . The azimuth angle rotation is identical to the extinction angle rotation [25].

It has been shown previously that irradiation of thin films of DR1-glass with a linearly polarized single beam at normal incidence results in the formation of SSRG consisting in a patchwork of domains with gratings along two distinct orientations and with a pitch comparable to the laser wavelength [34]. These gratings follow a first order stimulated Wood anomaly [35] in two directions determined by the optical anisotropy induced into the molecular glass [30]. However, for oblique irradiation under an incidence angle $\theta$, owing to the different wave-vector projections of the incident beam on the sample plane in different directions, crossed gratings following stimulated Wood anomalies with different pitches are obtained. The direction of the gratings still is determined by the optical anisotropy and the pitch of the gratings is determined by the phase matching conditions:

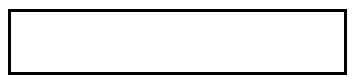

Where $K_{o} \sin \theta, K_{g x}$ and $K_{s x}$ are the component of the incident beam, the grating and the scattered beam wave-vector on the surface of the sample in the $\mathrm{x}$ direction respectively. If $\mathrm{K}_{\mathrm{g}}$ is parallel to $K_{0} \sin \theta$ and the scattered beam is at the grazing angle with the same phase as the incident wave, then the pitch of the gratings is given by:

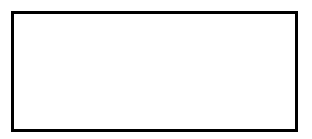

For $K_{g}$ perpendicular to the $K_{\mathrm{o}} \sin \theta$ the spacing is given by

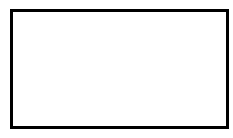


In consequence, under oblique irradiation of a beam polarized with a combination of $\mathrm{p}$ - and s-polarizations, crossed gratings with different pitches can form planar chiral structures. Fig. 1 shows AFM images of chiral SSRGs inscribed with both a linearly polarized beam at $45^{\circ}$ and with a right-handed circularly polarized light (RCPL) at an incident angle of $40^{\circ}$ and $-40^{\circ}$. Expectedly, the mirror images of these SSRGs could be inscribed with a linearly polarized beam at $-45^{\circ}$, and with a left-handed circularly polarized light (LCPL) at an incidence of $-40^{\circ}$ and $40^{\circ}$. The surfaces are chiral because both gratings with different pitches cross at an angle different from $90^{\circ}$, in a chevron-like arrangement. We see in Fig. 1a-1d that with linear light, one grating formed almost with $\mathrm{K}_{\mathrm{g}}$ perpendicular to the $K_{0} \sin \theta$ with about $560 \mathrm{~nm}$ pitch. A second grating formed with a pitch $\Lambda \approx 1200 \mathrm{~nm}$ almost along the $\mathrm{K}_{0} \sin \theta$ direction $\left(\theta=40^{\circ}\right)$, verifying equation (2). In the case of circular light in figures $1 \mathrm{e}-1 \mathrm{~h}$, one grating is oriented at about $40^{\circ}$ to the tilt, which projection is $\theta \approx 40^{\circ}$, in relative agreement with the grating pitch $\Lambda \approx 1000 \mathrm{~nm}$. We cannot distinguish any clear grating in the other direction, but mostly elongated dome-like structures. The Fourier transforms reveal their chirality.

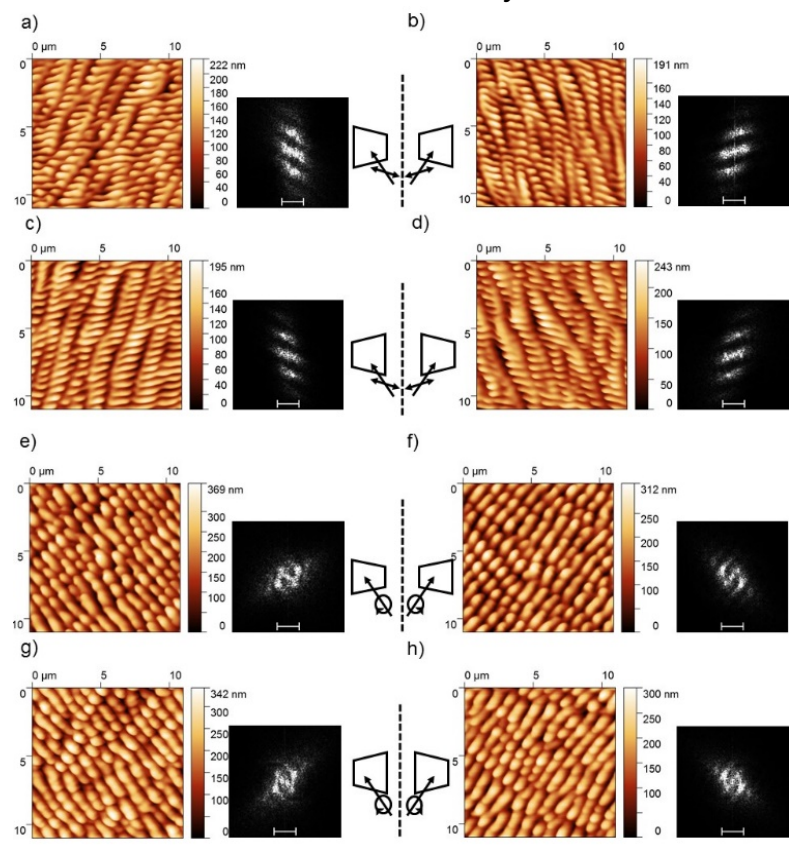

Fig. 1. AFM images of thin films of DR1-glass irradiated with a linearly and circularly polarized laser with $\lambda=532 \mathrm{~nm}$ and an irradiance of 300 $\mathrm{mW} / \mathrm{cm}^{2}$. Two-dimensional Fourier transforms are shown for each image. Light configuration with polarization direction and incidence angle in respect to the sample is included. SSRGs inscribed with a) $45^{\circ}$ linear polarization at $40^{\circ}$, b) $-45^{\circ}$ linear polarization at $-40^{\circ}$, c) $45^{\circ}$ linear polarization at $-40^{\circ}$, d) $-45^{\circ}$ linear polarization at $40^{\circ}$ e) right handed circular polarization at $40^{\circ} \mathrm{f}$ ) left handed circular polarization at $-40^{\circ} \mathrm{g}$ ) right handed circular polarization at $-40^{\circ}$ and $\mathrm{h}$ ) left-handed circular polarization at $40^{\circ}$ incidence angle. Scale bars on Fourier transforms are $2 \mu \mathrm{m}^{-1}$ long.

To evaluate the chirality of the gratings inscribed with different incidence angles, their optical activity was measured using the same setup as in figure 7 of [24]. Achiral gratings, for example a SSRG inscribed at normal irradiation, display no optical rotation. However, chiral SSRGs exhibit a noticeable change in the polarization azimuth of first-order-diffracted light. The first-orderdiffracted beam azimuth rotation as a function of initial polarization azimuth is reported in Fig. 2. The zero order transmitted beam did not show any azimuth rotation. The azimuth rotation of zero-order transmitted beam would reflect optical activity due to birefringence or 3D chirality [36].The absence of any optical activity at zero-order confirms that chirality is due to the planar chevron structures.
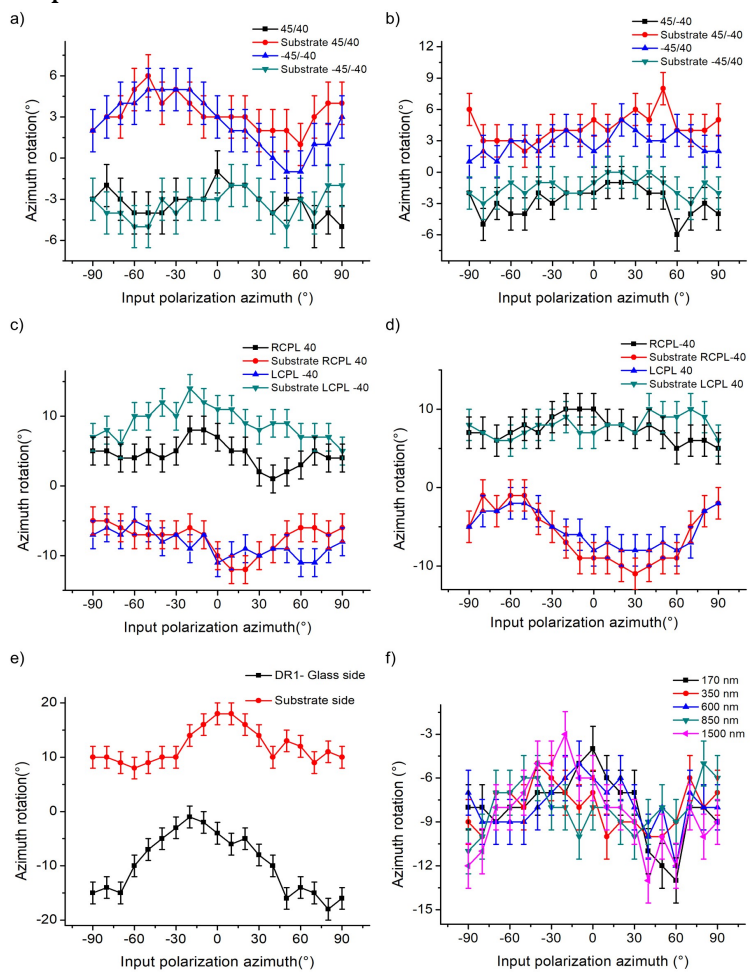

Fig. 2. Azimuth rotation as a function of initial polarization azimuth for SSRGs inscribed with polarization and incident angles of a) $45^{\circ}, 40^{\circ}$ b) $45^{\circ},-40^{\circ}$ c) RCPL, $40^{\circ}$ d) RCPL, $-40^{\circ}$, with their mirror images e) LCPL, $40^{\circ}$ silver coated. f) LCPL , $-40^{\circ}$ of different thicknesses (DR1glass side only).

Chiral SSRGs inscribed with linearly polarized light at $45^{\circ}$ and $-45^{\circ}$ displayed on average an azimuth rotation of $3^{\circ}$ (maximum $6^{\circ}$ ), whereas chiral SSRGs inscribed with a circularly polarized beam displayed on average an azimuth rotation of $8^{\circ}$ (maximum $10^{\circ}$ ). To verify the dependence of optical activity on propagation direction, the azimuth rotation was studied for probe beam illumination from both the DR1-glass side and the substrate side for both enantiomers. The recorded optical activity depends on the propagation direction of the beam, and it is reversed for the opposite propagation direction, thereby confirming that the gratings manifest $2 \mathrm{D}$ chiral properties. This demonstrates the nonreciprocity of the chiral effect. Ideally, the optical activity of a planar chiral structure should be perfectly inverted relative to that of its enantiomer, as well as relative to the orientation of the substrate side. However, SSRGs are not perfectly uniform structures. Consequently, there is a few degrees of discrepancy in optical activity between azo and substrate side irradiation. Also, as the SSRG growth process is subject to variance, SSRGs inscribed with inverted pump beam polarization and incidence angles are not perfect mirror images of each other (Fig. 1). As a result, the optical activities of two enantiomeric SSRG are not perfectly the inverse of each other. 
As mentioned above, the absence of azimuth rotation of the zeroorder transmitted beam suggests an absence of 3D chirality. In order to demonstrate that the optical activity is due to a surface grating and not a volume grating, a $40 \mathrm{~nm}$-thin layer of silver was deposited on top of the SSRG and the optical activity was studied on the silver-coated film. If the optical activity was due to birefringence or any other volume grating [37], the optical rotation of the polarization azimuth should then diminish. The Ag layer was deposited on the chiral SSRG inscribed with left-handed circular polarization at $40^{\circ}$ incidence. The optical activity of the coated film is shown in Fig. 2-e. Observation of optical activity before and after deposition of the Ag layer confirms the planar origin of the chirality which is contained in the chevron-like arrangement.

Sample thickness has been shown to impact tremendously on the effects of 3D chirality $[16,26,38]$. Samples with different thicknesses ranging from 150 to $1500 \mathrm{~nm}$ were prepared. The optical activity of SSRGs inscribed in these samples was studied (Fig. 2-f). No significant correlation between the recorded polarization rotation and sample thickness is observed. This is also a strong evidence of planar chirality, as opposed to 3D chirality, in the chevron-like structures observed in Fig. 1.

In order to understand the origin of the circular birefringence in chevron-like pavements, one must remember that SSRGs are printed like stimulated Wood anomalies in which the grating pitch is such that one of the diffraction orders, the 1st one in our experiment, is coupled into the plane of the film. In such circumstances, diffraction efficiency becomes strongly polarization-dependent, because in-plane diffraction is quantitative under s-polarization and vanishes under $\mathrm{p}$ polarization. Then, diffraction efficiency from the chevron structures exhibits the circular birefringence, which manifests as azimuth rotation in Fig. 2. These results are a confirmation of the optical nature of the spontaneous surface relief gratings [32].

In conclusion, planar chirality has been induced in spontaneous surface relief gratings (SSRGs) in thin films of an azobenzenefunctionalized molecular glass by direct chirality transfer from light. Irradiation of an azo film with a linearly polarized singlebeam laser source at normal incidence typically yields a patchwork of grating domains with an identical pitch but two grating orientations. By irradiating the sample with linearly polarized light or circularly polarized light at an oblique incidence angle, the resulting gratings show different pitches, thereby yielding crossed gratings that form chevron-like structures that show planar chirality, with azimuth rotations up to $10^{\circ}$. The bidimensional nature of the observed chirality, as well as the absence of tridimensional chirality, was confirmed. These findings highlight a method to generate non-reciprocal planar chiral objects by a simple irradiation process. More efficient and lossless chiral surfaces should find applications as optical isolators in nano-and micro-photonic devices.

Funding. The research reported herein was funded by the Canadian Defense Academy Academic Research Program (CDARP) and the National Science Research Council of Canada Discovery Grants (RGPIN-2015-05485) and CREATE program (Novel Chiral Materials: An International Effort in Research and Education).
1. N. Nilashis, Chirality in biological nanospaces: reactions in active sites, CRC Press, Boca Raton, FL, USA (2011)

2. C. Grande,N. H. Patel, Nature 457, 1007 (2009)

3. A. Guijarro, Y. Miguel, The origin of chirality in the molecules of life: a revision from awareness to the current theories and perspectives of this unsolved problem, RSC, London, UK (2008)

4. Y. Yang, R.C. Da Costa, M.J. Fuchter, A.J. Campbell, Nat. Photon. 7, 634 (2013)

5. L.V. Poulikakos, P. Gutsche, K.M. McPeak, S. Burger, J. Niegemann, C. Hafner, D.J. Norris, ACS Photon. 3, 1619 (2016)

6. A. Fazel Najafabadi, T. Pakizeh, ACS Photon. 4, 1447 (2017)

7. C.M. Soukoulis, M. Wegener, Nat. Photon. 5, 523 (2011)

8. L.C. Giancarlo, G.W. Flynn, Acc. Chem. Res. 33, 491 (2000)

9. V.W. Scarola, K. Park, S. Das Sarma, Phys. Rev. Lett. 93, 120503 (2004)

10. L. Poladian, M. Straton, Pure chiral optical fibres. Opt. Expr. 19, 968 (2011)

11. J.J.D. de Jong, L.N. Lucas, R.M. Kellogg, J.H. Van Esch, B.L. Feringa, Science 304, 278 (2004)

12. S. Zhang, Y.-S Park, J. Li, X. Lu, W. Zhang, X. Zhang, Phys. Rev. Lett. 102, 023901 (2009)

13. V.I. Kopp, J. Park, M. Wlodawski, J. Singer, D. Neugroschl, A.Z. Genack, J. Lightwave Technol. 32, 605 (2014)

14. A. Emoto, E. Uchida, T. Fukuda, Polymers 4, 150 (2012)

15. V.I. Kopp, V.M. Churikov, A.Z. Genack, Laser Focus World 44, 76 (2008)

16. E. Efrati , W. Irvine, Phys. Rev. X 4, 011003 (2014)

17. E. Plum, Univ. of Southampton (2010)

18. O. Arteaga, J. Sancho-Parramon, S. Nichols, B.M. Mazo, A. Canillas, S. Bosch, G. Markovich, B. Kahr, Opt. Expr. 24, 2242 (2016)

19. S.M. Fu, Y.K. Zhong, S.L. Yan, N.P. Ju, P.Y. Chen, M.-H. Kao, C.-H. Shen, J.-M. Shieh, Y.-C. Lai, P. Yu, A. Lin, IEEE 42nd Photovolt. Spec. Conf. , DOI: 10.1109/PVSC.2015.7356119 (2015)

20. P. Lodahl, S. Mahmoodian, S. Stobbe, A. Rauschenbeutel, P. Schneeweiss, J. Volz, H. Pichler, P. Zoller, Nature 541, 473 (2017)

21. Z. Fan, and A. O. Govorov, Nano Lett. 10, 2580 (2010)

22. A. Papakostas, A. Potts, D.M. Bagnall, N.I. Zheludev, Phys. Rev. Lett. 90 107404 (2003)

23. A. Potts, W. Zhang, D.M. Bagnall, Phys. Rev. A 77, 043816 (2008)

24. Y. Kim, N. Tamaoki, J. Mater. Chem. C 2, 9258 (2014)

25. A. Ahmadi Kandjani, R. Barille, J.-M. Nunzi, R. Kheradmand, H. Tajalli, Phys. Stat. Sol. C 8, 2773 (2011)

26. Z. Sekkat, W. Knoll, Photoreactive organic thin films, Academic Press: San Diego, CA, USA (2002)

27. A. Ambrosio, L. Marrucci, F. Borbone, A. Roviello, P. Maddalena, Nat. Commun. 3, 989 (2012)

28. H. Sumimura, T. Fukuda, J.Y. Kim, D. Barada, M. Itoh, T. Yatagai, Jpn. J. Appl. Phys. 45, 451 (2006)

29. A. Natansohn, P. Rochon, Chem. Rev. 102, 4139 (2002)

30. C.J. Barrett, J.I. Mamiya, K.G. Yager, T. Ikeda, Soft Matter 3, 1249 (2007)

31. C. Hubert, C. Fiorini-Debuisschert, L. Rocha, P. Raimond, J.-M. Nunzi, J. Opt. Soc. Am. B 24, 1839(2007)

32. L. Mazaheri, R.G. Sabat, O. Lebel, J.-M. Nunzi, Opt. Mater. 62, 378 (2016)

33. R. Kirby, R.G. Sabat, J.-M. Nunzi, O. Lebel, J. Mater. Chem. C 2, 841 (2014)

34. L. Mazaheri, S. Rao Bobbara, O. Lebel, J.-M. Nunzi, Opt. Lett. 41, 2958 (2016)

35. A.E. Siegman, P.E. Fauchet, IEEE J. Quantum Electron. 22, 1384 (1986)

36. M. Christoph, C. Rockstuhl, and F. Lederer, Phys. Rev. A 82.5 053811(2010)

37. K. G. Yager, C. Barrett, J. chem. phys. 1 094908(2007)

38. M. Hentschel, M. Schäferling, X. Duan, H. Giessen, N. Liu, Sci. Adv. 3, 1602735 (2017)

\section{References}




\section{References}

1. N. Nilashis, Chirality in biological nanospaces: reactions in active sites, CRC Press, Boca Raton, FL, USA (2011)

2. C. Grande,N. H. Patel, Nodal signalling is involved in left-right asymmetry in snails, Nature 457, 1007 (2009)

3. A. Guijarro, Y. Miguel, The origin of chirality in the molecules of life: a revision from awareness to the current theories and perspectives of this unsolved problem, RSC, London, UK (2008)

4. Y. Yang, R.C. Da Costa, M.J. Fuchter, A.J. Campbell, Circularly polarized light detection by a chiral organic semiconductor transistor, Nat. Photon. 7, 634 (2013)

5. L.V. Poulikakos, P. Gutsche, K.M. McPeak, S. Burger, J. Niegemann, C. Hafner, D.J. Norris, Optical chirality flux as a useful far-field probe of chiral near fields, ACS Photon. 3, 1619 (2016)

6. A. Fazel Najafabadi, T. Pakizeh, Analytical chiroptics of the 2D and 3D nanoantennas, ACS Photon. 4, 1447 (2017)

7. C.M. Soukoulis, M. Wegener, Past achievements and future challenges in the development of three-dimensional photonic metamaterials, Nat. Photon. 5, 523 (2011)

8. L.C. Giancarlo, G.W. Flynn, Raising flags: applications of chemical marker groups to study self-assembly, chirality, and orientation of interfacial films by scanning tunneling microscopy, Acc. Chem. Res. 33, 491 (2000)

9. V.W. Scarola, K. Park, S. Das Sarma, Chirality in quantum computation with spin cluster qubits, Phys. Rev. Lett. 93, 120503 (2004)

10. L. Poladian, M. Straton, Pure chiral optical fibres, Pure chiral optical fibres. Opt. Expr. 19, 968 (2011)

11. J.J.D. de Jong, L.N. Lucas, R.M. Kellogg, J.H. Van Esch, B.L. Feringa, Reversible optical transcription of supramolecular chirality into molecular chirality, Science 304, 278 (2004)

12. S. Zhang, Y.-S Park, J. Li, X. Lu, W. Zhang, X. Zhang, Negative refractive index in chiral metamaterials, Phys. Rev. Lett. 102, 023901 (2009)

13. V.I. Kopp, J. Park, M. Wlodawski, J. Singer, D. Neugroschl, A.Z. Genack, Chiral fibers: microformed optical waveguides for polarization control, sensing, coupling, amplification, and switching J. Lightwave Technol. 32, 605 (2014)

14. A. Emoto, E. Uchida, T. Fukuda, Optical and physical applications of photocontrollable materials: Azobenzene-containing and liquid crystalline polymers, Polymers 4, 150 (2012)

15. V.I. Kopp, V.M. Churikov, A.Z. Genack, Fiber Sensing - Chiral-fiber gratings sense the environment, Laser Focus World 44, 76 (2008)

16. E. Efrati , W. Irvine, Orientation dependent handedness and chiral design, Phys. Rev. X 4, 011003 (2014)

17. E. Plum, Chirality and metamaterials, Univ. of Southampton (2010)

18. O. Arteaga, J. Sancho-Parramon, S. Nichols, B.M. Mazo, A. Canillas, S. Bosch, G. Markovich, B. Kahr, Relation between 2D/3D chirality and the appearance of chiroptical effects in real nanostructures, Opt. Expr. 24, 2242 (2016)

19. S.M. Fu, Y.K. Zhong, S.L. Yan, N.P. Ju, P.Y. Chen, M.-H. Kao, C.-H. Shen, J.-M. Shieh, Y.-C. Lai, P. Yu, A. Lin, Non-reciprocal meta-surfaces for nanophotonic light trapping: optical isolators versus solar cells, IEEE 42nd Photovolt. Spec. Conf. , DOI: 10.1109/PVSC.2015.7356119 (2015)

20. P. Lodahl, S. Mahmoodian, S. Stobbe, A. Rauschenbeutel, P. Schneeweiss, J. Volz, H. Pichler, P. Zoller, Chiral quantum optics, Nature 541, 473 (2017)

21. Z. Fan, and A. O. Govorov, Plasmonic circular dichroism of chiral metal nanoparticle assemblies, Nano Lett. 10, 2580 (2010)

22. A. Papakostas, A. Potts, D.M. Bagnall, N.I. Zheludev, Optical manifestations of planar chirality, Phys. Rev. Lett. 90, 107404 (2003)

23. A. Potts, W. Zhang, D.M. Bagnall, Nonreciprocal diffraction through dielectric gratings with two-dimensional chirality, Phys. Rev. A 77, 043816 (2008)
24. Y. Kim, N. Tamaoki, J. Mater. A photoresponsive planar chiral azobenzene dopant with high helical twisting power, Chem. C 2, 9258 (2014)

25. A. Ahmadi Kandjani, R. Barille, J.-M. Nunzi, R. Kheradmand, H. Tajalli, Light induced 2D chiral structure on the surface of azo-polymer films, Phys. Stat. Sol. C 8, 2773 (2011)

26. Z. Sekkat, W. Knoll, Photoreactive organic thin films, Academic Press: San Diego, CA, USA (2002)

27. A. Ambrosio, L. Marrucci, F. Borbone, A. Roviello, P. Maddalena, Lightinduced spiral mass transport in azo-polymer films under vortex-beam illumination, Nat. Commun. 3, 989 (2012)

28. H. Sumimura, T. Fukuda, J.Y. Kim, D. Barada, M. Itoh, T. Yatagai, Photoinduced chirality in azobenzene amorphous copolymer bearing large birefringent moiety, Jpn. J. Appl. Phys. 45, 451 (2006)

29. A. Natansohn, P. Rochon, Photoinduced motions in azo-containing polymers, Chem. Rev. 102, 4139 (2002)

30. C.J. Barrett, J.I. Mamiya, K.G. Yager, T. Ikeda, Photo-mechanical effects in azobenzene-containing soft materials, Soft Matter 3, 1249 (2007)

31. C. Hubert, C. Fiorini-Debuisschert, L. Rocha, P. Raimond, J.-M. Nunzi, Spontaneous photoinduced patterning of azo-dye polymer films: the facts, J. Opt. Soc. Am. B 24, 1839(2007)

32. L. Mazaheri, R.G. Sabat, O. Lebel, J.-M. Nunzi, Unraveling the nucleation and growth of spontaneous surface relief gratings with thin films of Disperse Red 1 glass, Opt. Mater. 62, 378 (2016)

33. R. Kirby, R.G. Sabat, J.-M. Nunzi, O. Lebel, Disperse and disor-dered: a mexylaminotriazine-substituted azobenzene derivative with superior glass and surface relief grating formation, J. Mater. Chem. C 2, 841 (2014)

34. L. Mazaheri, S. Rao Bobbara, O. Lebel, J.-M. Nunzi, Photoinduction of spontaneous surface relief gratings on Azo DR1 glass, Opt. Lett. 41, 2958 (2016)

35. A.E. Siegman, P.E. Fauchet, Stimulated Wood's anomalies on laserilluminated surfaces, IEEE J. Quantum Electron. 22, 1384 (1986)

36. M. Christoph, C. Rockstuhl, and F. Lederer, Advanced Jones calculus for the classification of periodic metamaterials, Phys. Rev. A 82.5 053811(2010)

37. K. G. Yager, C. Barrett, Confinement of surface patterning in azopolymer thin films, J. chem. phys. 1 094908(2007)

38. M. Hentschel, M. Schäferling, X. Duan, H. Giessen, N. Liu, Chiral plasmonics, Sci. Adv. 3, 1602735 (2017) 